\title{
Evidence in Favor of the Baez-Duarte Criterion for the Riemann Hypothesis
}

\author{
Marek Wolf \\ Institute of Theoretical Physics, University of Wroctaw \\ Pl. Maxa Borna 9, PL-50-204 Wroctaw, Poland \\ e-mail:mwolf@ift.uni.wroc.pl
}

(Received; 22 October 2008; published online: 30 October 2008 )

\begin{abstract}
We present the results of the numerical experiments in favor of the Baez-Duarte criterion for the Riemann Hypothesis. We give formulae allowing calculation of numerical values of the numbers $c_{k}$ appearing in this criterion for arbitrary large $k$. We present plots of $c_{k}$ for $k \in\left(1,10^{9}\right)$.
\end{abstract}

Key words: Riemann Hypothesis, Baez-Duarte criterion for RH

\section{INTRODUCTION}

In 1997 K. Maślanka [1] proposed a new formula for the zeta Riemann function valid on the whole complex plane $\mathbb{C}$ except a point $s=1$ :

$$
\zeta(s)=\frac{1}{1-s} \sum_{k=0}^{\infty} \frac{\Gamma\left(k+1-\frac{s}{2}\right)}{\Gamma\left(1-\frac{s}{2}\right)} \frac{A_{k}}{k !}
$$

where coeffcients $A_{k}$ are given by

$$
A_{k}=\sum_{j=0}^{k}\left(\begin{array}{l}
k \\
j
\end{array}\right)(2 j-1) \zeta(2 j+2) \text {. }
$$

This formula was rigorously proven by L. Baez-Duarte in 2003 [2]. In the subsequent preprint [3] the same author proved the new criterion for the Riemann Hypothesis, while its journal version appeared two years later [4]. The Riemann Hypothesis (RH) states that the nontrivial zeros $\rho$ of the function $\zeta(s)$ have the real part equal $\mathfrak{R}(\rho)=1 / 2$. Although Riemann did not request it, today it is often additionally demanded that zeros on the critical line $\mathfrak{R}(s)=1 / 2$ should be simple. Baez-Duarte considered the sequence of numbers $c_{k}$ defined by:

$$
c_{k}=\sum_{j=0}^{k}(-1)^{j}\left(\begin{array}{l}
k \\
j
\end{array}\right) \frac{1}{\zeta(2 j+2)} .
$$

He proved that $\mathrm{RH}$ is equivalent to the following rate of decreasing to zero of the above sequence:

$$
c_{k}=\mathscr{O}\left(k^{-3 / 4+\varepsilon}\right) \text { for each } \varepsilon>0 .
$$

Furthermore, if $\varepsilon$ can be put zero, i.e. if $c_{k}=\boldsymbol{O}\left(k^{-3 / 4}\right)$, then the zeros of $\zeta(s)$ are simple. Baez-Duarte also proved in [4] that it is not possible to replace $3 / 4$ by $3 / 4+\varepsilon$.

Neither in [4] nor in [6] is it explicitly written whether the sequence $c_{k}$ starts from $k=0$ or $k=1$. However in [4] a few formulas contain $k=0$, i.e. summation starts from $c_{0}$. The point is that if we allow $k=0$, for which $c_{0}=6 / \pi^{2}$, then the inversion formula (see e.g. [7]) is fulfilled:

$$
\frac{1}{\zeta(2 k+2)}=\sum_{j=0}^{k}(-1)^{j}\left(\begin{array}{c}
k \\
j
\end{array}\right) c_{j} .
$$

However, we see no application of the above formula, except for a possibility of checking some of the statements made in [13]. Furthermore, if the Baez-Duarte sequence $c_{k}$ starts from $k=0$ then the following identity holds:

$$
\sum_{k=0}^{\infty} \frac{c_{k} x^{k}}{k !}=e^{x} \sum_{k=0}^{\infty} \frac{(-1)^{k} x^{k}}{k ! \zeta(2 k+2)} .
$$

It is an application of the general formal identity:

$$
\sum_{k=0}^{\infty}\left(\sum_{j=0}^{k}\left(\begin{array}{l}
k \\
j
\end{array}\right) a_{j}\right) \frac{x^{k}}{k !}=e^{x} \sum_{k=0}^{\infty} \frac{c_{k} x^{k}}{k !},
$$


where $a_{k}$ should not increase too fast with $k$ to ensure convergence of series ${ }^{1}$. Putting here $a_{j}=(-1)^{j} b_{j}$ gives the usual formula appearing in the finite difference theory (see [14] §1):

$$
\sum_{k=0}^{\infty}\left(\sum_{j=0}^{k}(-1)^{j}\left(\begin{array}{l}
k \\
j
\end{array}\right) b_{j}\right) \frac{x^{k}}{k !}=e^{x} \sum_{k=0}^{\infty} \frac{(-1)^{k} b_{k} x^{k}}{k !} .
$$

The identity (4) can be used to establish the connection with the Riesz criterion for RH (original paper [9], discussed in $[4,11])$. Riesz has considered the function:

$$
\begin{aligned}
R(x) & =\sum_{k=1}^{\infty} \frac{(-1)^{k+1} x^{k}}{(k-1) ! \zeta(2 k)}= \\
= & \sum_{k=0}^{\infty} \frac{(-1)^{k} x^{k+1}}{k ! \zeta(2 k+2)} .
\end{aligned}
$$

Unconditionally it can be proved that $R(x)=\boldsymbol{O}\left(x^{1 / 2+\varepsilon}\right)$, see [8] $\$ 14.32$. Riesz has proved that the Riemann Hypothesis is equivalent to slower increasing of the function $R(x)$ :

$$
R H \Leftrightarrow R(x)=\sigma\left(x^{1 / 4+\varepsilon}\right)
$$

But from (4) we get:

$$
\sum_{k=0}^{\infty} \frac{c_{k} x^{k}}{k !}=\frac{e^{x}}{x} R(x)
$$

thus the generating function for $c_{k}$ can be expressed by $R(x)$. In [10] it is proven that for any real number $\delta>-3 / 2$ we have

$$
R(x)=O\left(x^{\delta+1}\right) \Leftrightarrow c_{k}=O\left(k^{\delta}\right)
$$

The proof is based on the relation $R(k) / k \approx c_{k}$.

\section{COMPUTER EXPERIMENTS}

The criterion (2) seemed to be very well suited for computer verification. At the end of [3] Baez-Duarte wrote a sentence "A test for the first $c_{k}$ up to $k=1000$ shows a very pleasant smooth curve". However, for larger values of $k$ the true behavior of the sequence turned out to be more complicated: instead of monotonic tending to zero there appeared oscillations and $c_{k}$ changed the sign at first

\footnotetext{
${ }^{1}$ Indeed, collecting on the 1.h.s. terms multiplying $a_{j}$ we get: $a_{j} \sum_{k=j}^{\infty}\left(\begin{array}{c}k \\ j\end{array}\right) \frac{x^{k}}{k !}=a_{j} \sum_{k=j}^{\infty} \frac{k !}{j !(j-k) !} \frac{x^{k}}{k !}=a_{j} \frac{x^{j}}{j !} \sum_{n=0}^{\infty} \frac{x^{n}}{n !}=a_{j} \frac{x^{j}}{j !} e^{x}$ and summing over $j$ gives r.h.s.
}

for $^{2} k=19320: c_{19319}=-1.7870567 \times 10^{-13}$ while $c_{19320}=$ $9.170232808 \ldots \times 10^{-12}$. The next sign change is: $c_{22526}=$ $2.2292905301 \ldots \times 10^{-13}$ but $c_{22527}=-6.5057526 \times 10^{-12}$.

To our knowledge, the first plot of $c_{k}$ for $k$ up to 95000 appeared in the book [5] published in Polish. The same plot was reproduced in [4]. Data used to make this plot consisted of $c_{k}$ calculated every 500-th $k$-it is very time consuming to get $c_{k}$ directly from (1). Indeed, for large $j$ the values of $\zeta(2 j$ +2 ) very quickly become practically equal to 1 , thus the summation of alternating series gives wrong results when not performed with a sufficient number of digits accuracy. For example, Table 1 presents values of the partial sums for $c_{12000}$ recorded every thousand summands (the calculation was performed with precision of 9000 digits).

Table 1.

\begin{tabular}{rc}
\hline$n$ & $\sum_{j=0}^{n}(-1)^{j}\left(\begin{array}{c}12000 \\
j\end{array}\right) \frac{1}{\zeta(2 j+2)}$ \\
\hline 1000 & $8.6575528427959311728 \times 10^{1492}$ \\
2000 & $1.0610772171540382076 \times 10^{2346}$ \\
3000 & $2.6820721693716011525 \times 10^{2928}$ \\
4000 & $8.4511383022435967124 \times 10^{3314}$ \\
5000 & $1.8751018390471552047 \times 10^{3537}$ \\
6000 & $8.3417729099514988532 \times 10^{3609}$ \\
7000 & $1.3393584564622537177 \times 10^{3537}$ \\
8000 & $4.2255691511217983562 \times 10^{3314}$ \\
9000 & $8.9402405645720038417 \times 10^{2927}$ \\
10000 & $2.1221544343080764152 \times 10^{2345}$ \\
11000 & $7.8705025843599374298 \times 10^{1491}$ \\
12000 & $-1.6973092190852083930 \times 10^{-7}$ \\
\hline
\end{tabular}

Let us remark that the partial sums for $n$ and 12000- $n$ are of the same order. The binomial coeficients become very large numbers in the middle and to get accurate value of $c_{k}$ one needs a lot of digits accuracy during the calculation. Maślanka has used Mathematica to perform these calculation. Over three years ago we started to calculate $c_{k}$ using the free package PARI/GP [12] developed especially for number theoretical purposes, which allows practically arbitrary accuracy arithmetics both fixed-point as well as floating-point. We started to calculate consecutive $c_{k}$ for each $k$ with the help of the following script in Pari:

\footnotetext{
2 in fact if the sequence $c_{k}$ starts from $k=0$ the first sign change occurs for $c_{0}=6 / \pi^{2}>0$ and $c_{1}=6 / \pi^{2}-90 / \pi^{4}=\left(6 \pi^{2}-90\right) / \pi^{4} \approx$ $\approx\left(-30 / \pi^{4}\right)<0$ (more precisely $c_{1}=-0.3160113011 \ldots$ )
} 


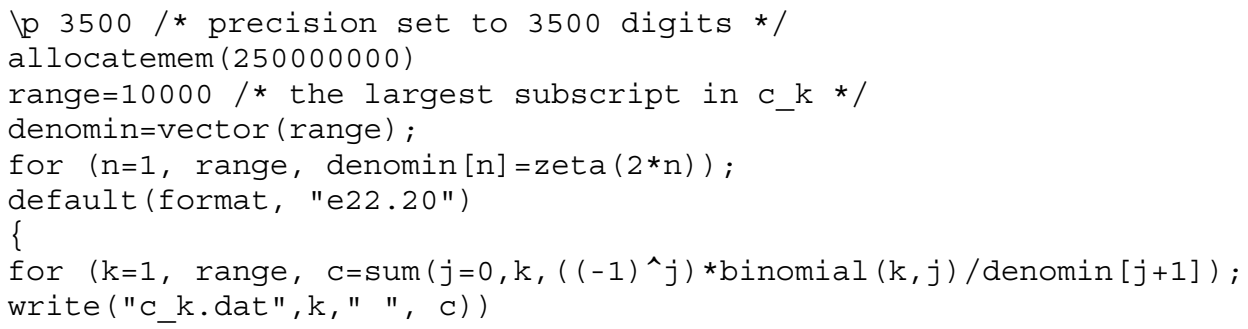

The problem encountered during these calculations was that it seemed not possible to change the accuracy of calculation while running the script (the command $\mid p 3500$ above). Thus we had to change the precision by hand. It turned out that when the precision was to small, produced values of $c_{k}$ were completely wrong, differing from the correct ones by ten to a very large power. The rule learned from these examples for precision set to make calculations confident was that the number of digits should be at least enough to distinguish between 1 and $1+1 / 2^{k}$ in the zeta appearing in (1), i.e. the precision set to calculate $c_{k}$ should be at least $\mid \mathrm{p}=k * \log _{10}(2)$. Table 2 presents the real example we saw during the calculations: when the precision was set to 60000 digits the values of $c_{k}$ for $k$ between 198000 and 200000 were $\left(198000 \times \log _{10}(2)=\right.$ 59603.93914, $\left.200000 \times \log _{10}(2)=60205.99913\right):$

Table 2.

\begin{tabular}{cc}
\hline$k$ & $c_{k}$ \\
\hline 198000 & $-8.1809420017968747912 \times 10^{-9}$ \\
198500 & $-8.1130397250007379108 \times 10^{-9}$ \\
199000 & $-8.0431163120575296823 \times 10^{-9}$ \\
199500 & $3.4122583912205353616 \times 10^{49}$ \\
200000 & $-1.9276608381598523688 \times 10^{200}$ \\
\hline
\end{tabular}

Maślanka kindly sent me the values of $c_{k}$ from his calculations up to $k=95000$ with $k$ jumping in intervals of 500 , i.e. $k=500 l$. In autumn 2005 , I started to continue working on the cluster of 8 processors Xeon $2.8 \mathrm{GHz}$, with 4 GB RAM per node of two processors ${ }^{3}$, with the aim to reach $k=200000$ also every 500-th value of $k$ using PARI/GP computer algebra system [12]. Over the five months of computations I have used between 4 and 6 processors to calculate $c_{k}$ in different intervals of $k$. When these calculations were running I found the paper [4] where

\footnotetext{
${ }^{3}$ Because we have used 32-bits version of PARI/GP I was able to use $2^{\wedge} 31$ bytes $=2$ GB of RAM per process.
}

not assume the validity of Riemann Hypothesis in contrast to formulae presented by Maślanka or below. Using these formulae $c_{k}$ can be calculated very quickly for practically arbitrary $k-$ it is very time-consuming to calculate $c_{k}$ without assuming RH.

\section{EXPLICIT FORMULAE}

The formulae presented in [4] and [6] expressing $c_{k}$ directly in terms of the zeros of $\zeta(s)$ are essentially the same, they differ in the manner they were derived. Maślanka has used the binomial transforms discussed in [14] while Baez-Duarte is developing the whole machinery by himself. The formulae of these two authors can be written as a sum of two parts: quickly decreasing with $k$ trend $\bar{c}_{k}$ and oscillations $\tilde{c}_{k}$ :

$$
c_{k}=\bar{c}_{k}+\tilde{c}_{k}
$$

where:

$$
\bar{c}_{k}=-\frac{1}{(2 \pi)^{2}} \sum_{m=2}^{\infty} \frac{B(k+1, m)}{\Gamma(2 m-1)} \frac{(-1)^{m}(2 \pi)^{2 m}}{\zeta(2 m-1)}
$$

and oscillating part:

$$
\begin{aligned}
\tilde{c}_{k} & =\sum_{\rho} \frac{\Gamma(k+1) \Gamma\left(\frac{1+\rho}{2}\right)}{\Gamma\left(k+1+\frac{1+\rho}{2}\right)} \frac{1}{\zeta^{\prime}(1-\rho)}= \\
& =\sum_{\rho} B\left(k+1, \frac{1+\rho}{2}\right) \frac{1}{\zeta^{\prime}(1-\rho)}
\end{aligned}
$$

where it is assumed that zeros of $\zeta(s)$ are simple: $\zeta^{\prime}(\rho) \neq 0$ and the sum is over all (i.e. on the positive as well as negative imaginary axis) nontrivial zeros of the $\zeta(s)$, i.e. $\zeta(\rho)=0$ and $\Im \rho \neq 0$ and

$$
B(w, z)=\frac{\Gamma(w) \Gamma(z)}{\Gamma(w+z)}
$$


is the Beta function. In fact, Baez-Duarte is skipping the trend remarking only that it is of the order $o(1 / k)$ (Remark 1.6 in [4]). Theoretically the formula for $\tilde{c}_{k}$ is valid in the limit of large $k$, but surprisingly the numbers produced from the above formulae (10) and (11) are practically the same as obtained from the generic formula (1) for all $k$, e.g. already for $k=2$ we get $c_{2}=-0.25699711$ from (1), while (10) and (11) give $c_{2}=-0.256969863$ and accuracy increases with $k$. It suggests that the integrals $J_{k}$ appearing in [4] in the proof of the Theorem 1.5 are decreasing to zero rather fast with $k$.

First let us consider the trend. It can be calculated directly from (10):

$$
\begin{gathered}
\bar{c}_{k}=-\frac{1}{(2 \pi)^{2}} \times \\
\times \sum_{m-2}^{\infty} \frac{1}{(k+1)(k+2) \ldots(k+m) m(m+1) \ldots(2 m-2)} \frac{(-1)^{m}(2 \pi)^{2 m}}{\zeta(2 m-1)}
\end{gathered}
$$

thus we have

Using this formula we were able to produce every 500-th value of $\bar{c}_{k}$ for $k=500,1000, \ldots 10^{9}$ performing calculations in Pari with 100 digits accuracy in about 4 hours. For large $k$ we have used the following asymptotic expansion of (12):

$$
\begin{gathered}
\bar{c}_{k}=\frac{1}{(k+1)(k+2)} \times \\
\times\left(\frac{(2 \pi)^{2}}{2 \zeta(3)}-\frac{(2 \pi)^{4}}{12(k+3) \zeta(5)}+\frac{(2 \pi)^{6}}{120(k+3)(k+4) \zeta(7)}\right) .
\end{gathered}
$$

It can be further simplified to:

$$
B(a, x) \sim x^{-a} \Gamma(a) \quad \text { for } x \text { large. }
$$

Using it for large $k$ and assuming the Riemann Hypothesis:

$$
\bar{c}_{k}=-\frac{1}{k^{2}} \frac{(2 \pi)^{2}}{2 \zeta(3)} .
$$

The comparison of these formulae is given in Table 3.

Now we consider the oscillating part $\tilde{c}_{k}$. Since PARI/GP does not have built in $B(x, y)$ function, we had to use the $\Gamma(z)$ functions instead. Because of the fast growth of the $\Gamma(x)$ function even in PARI/GP it was not possible to pursue with formula (11) for large $k$. Namely it crashes for $k=356000$ because of overflow. But there is the following asymptotic formula (see e.g. [15], §1.8.7):

$$
\frac{\Gamma(x)}{\Gamma(x+a)} \sim x^{-a}, \quad x \rightarrow \infty
$$
$\rho_{l}=1 / 2+i \gamma_{l}, \bar{\rho}_{l}=1 / 2-i \gamma_{l}\left(=1-\rho_{l}\right)$ after collecting together in pairs conjugate zeros we get:

$$
\begin{gathered}
\tilde{c}_{k}=\frac{2}{(k+1)^{3 / 4}} \times \\
\times \sum_{l=1}^{\infty} \alpha_{l} \cos \left(\frac{1}{2} \gamma_{l} \log (k+1)\right)-\beta_{l} \sin \left(\frac{1}{2} \gamma_{l} \log (k+1)\right)
\end{gathered}
$$

where I have denoted:

$$
\alpha_{l}=\Re\left(\frac{\Gamma\left(\frac{1+\rho_{l}}{2}\right)}{\zeta^{\prime}\left(\bar{\rho}_{l}\right)}\right),
$$

Table 3.

\begin{tabular}{cccc}
\hline$k$ & $\bar{c}_{k}$ from Eq. (12) & $\bar{c}_{k}$ from Eq. (13) & $\bar{c}_{k}$ from Eq. (14) \\
\hline 1 & $-2.60052406393 \times 10^{-1}$ & $-4.0752814729 \times 10^{3}$ & $-1.6421193331 \times 10^{1}$ \\
10 & $-6.9069591105 \times 10^{-2}$ & $-2.0455052855 \times 10^{1}$ & $-1.6421193331 \times 10^{-1}$ \\
$10^{2}$ & $-1.4804264464 \times 10^{-3}$ & $-5.9943727867 \times 10^{-3}$ & $-1.6421193331 \times 10^{-3}$ \\
$10^{3}$ & $-1.6248041420 \times 10^{-5}$ & $-1.6824923096 \times 10^{-5}$ & $-1.6421193331 \times 10^{-5}$ \\
$10^{4}$ & $-1.6403755367 \times 10^{-7}$ & $-1.6418022398 \times 10^{-7}$ & $-1.6421193331 \times 10^{-7}$ \\
$10^{5}$ & $-1.6419448299 \times 10^{-9}$ & $-1.6420436474 \times 10^{-9}$ & $-1.6421193331 \times 10^{-9}$ \\
$10^{6}$ & $-1.6421018816 \times 10^{-11}$ & $-1.6421113244 \times 10^{-11}$ & $-1.6421193331 \times 10^{-11}$ \\
$10^{7}$ & $-1.6421175880 \times 10^{-13}$ & $-1.6421185279 \times 10^{-13}$ & $-1.6421193331 \times 10^{-13}$ \\
$10^{8}$ & $-1.6421191586 \times 10^{-15}$ & $-1.6421192526 \times 10^{-15}$ & $-1.6421193331 \times 10^{-15}$ \\
$10^{9}$ & $-1.6421193157 \times 10^{-17}$ & $-1.6421193251 \times 10^{-17}$ & $-1.6421193331 \times 10^{-17}$ \\
\hline
\end{tabular}




$$
\beta_{l}=\mathfrak{I}\left(\frac{\Gamma\left(\frac{1+\rho_{l}}{2}\right)}{\zeta^{\prime}\left(\bar{\rho}_{l}\right)}\right) .
$$

In (16) the decreasing of $c_{k}$ like $k^{-3 / 4}$ is obtained as an overall amplitude of the "waves" composed of the cosines and sines with the "frequencies" proportional to imaginary parts of the nontrivial zeros of $\zeta(s)$. The coeffcients $\alpha_{l}$ and $\beta_{l}$ decrease to zero very fast with $l$. Namely using the Hadamard product for $\zeta(s)$ :

$$
\zeta(s)=\frac{(2 \pi)^{s} e^{-(1+C / 2) s}}{2(s-1) \Gamma(s / 2+1)} \prod_{\rho}\left(1-\frac{s}{\rho}\right) e^{s / \rho},
$$

where $C=0.57721566490153286 \ldots$ is the Euler constant, the derivative of $\zeta(s)$ at zeros can be computed. Taking into account miraculous simplifications, $\rho \rho_{l}+\bar{\rho} \rho_{l}=\rho_{l}$ and the identity

$$
\Gamma(1+z) \Gamma(1-z)=\frac{\pi z}{\sin (\pi z)}
$$

I have obtained that:

$$
\left|\alpha_{l}\right| \propto e^{-\pi \gamma_{l} / 4}, \quad\left|\beta_{l}\right| \propto e^{-\pi \gamma_{l} / 4}
$$

Because imaginary parts of zeros take large values, it suffices to sum in (16) over a few first zeros. I have used 10 zeros and Table IV gives coefficients $\alpha_{l}$ and $\beta_{l}$ and comparison with (19).

However, calculations with the first zero $\gamma_{1}=$ $14.13472514173469 \ldots$ give numbers which differ much less than $1 \%$ (see $l=1$ and $l=2$ in Table IV) from those calculated with a larger number of terms in (16) as well

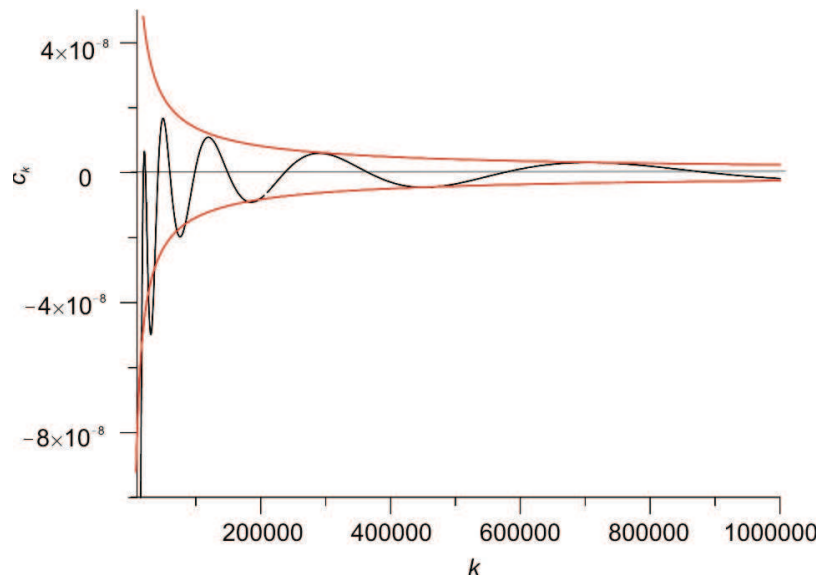

Fig. 1. The plot of $c_{k}$ for $k \in\left(1,10^{6}\right)$. At $k=200000$ a small gap is visible to distinguish between $c_{k}$ calculated from generic formula (1) and from explicit formulas presented in Sec. 3

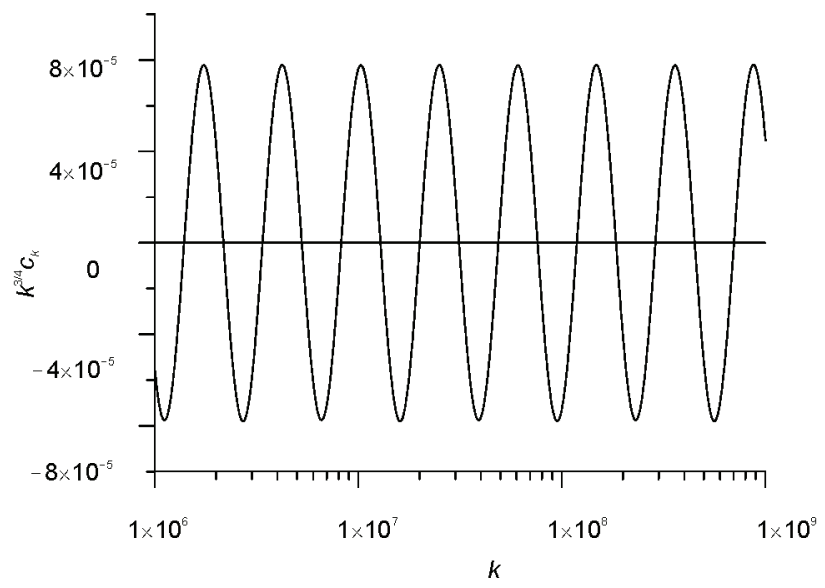

Fig. 2. The plot of $k^{3 / 4} c_{k}$ for $k \in 2\left(10^{6}, 10^{9}\right)$

Table 4

\begin{tabular}{cccc}
\hline & $\alpha_{l}$ & $\beta_{l}$ & $e^{-\pi \varphi_{l} / 4}$ \\
\hline 1 & $2.029173866 \times 10^{-5}$ & $-3.315924256 \times 10^{-5}$ & $1.50914 \times 10^{-5}$ \\
2 & $-3.333265938 \times 10^{-8}$ & $-1.298336420 \times 10^{-7}$ & $6.75315 \times 10^{-8}$ \\
3 & $2.886139424 \times 10^{-9}$ & $-4.153918097 \times 10^{-9}$ & $2.94404 \times 10^{-9}$ \\
4 & $4.813880001 \times 10^{-11}$ & $-6.332017430 \times 10^{-11}$ & $4.19039 \times 10^{-11}$ \\
5 & $7.546769513 \times 10^{-12}$ & $7.526891498 \times 10^{-12}$ & $5.83506 \times 10^{-12}$ \\
6 & $6.162524600 \times 10^{-14}$ & $1.942118979 \times 10^{-13}$ & $1.51209 \times 10^{-13}$ \\
7 & $-1.578482027 \times 10^{-14}$ & $1.184829593 \times 10^{-14}$ & $1.10374 \times 10^{-14}$ \\
8 & $-1.071381892 \times 10^{-15}$ & $-2.209146437 \times 10^{-15}$ & $1.66491 \times 10^{-15}$ \\
9 & $9.328038737 \times 10^{-19}$ & $-7.472197226 \times 10^{-17}$ & $4.22403 \times 10^{-17}$ \\
10 & $1.747829093 \times 10^{-17}$ & $1.122667624 \times 10^{-17}$ & $1.05303 \times 10^{-17}$ \\
\hline
\end{tabular}


as with $c_{k}$ for $k<200000$ obtained directly from (1) without assuming RH. The plots of $c_{k}$ for $k$ up to $10^{9} \mathrm{ob}-$ tained from those formulae are given in the Fig. 1 and Fig. 2. In Fig. 2 there is a logarithmic $k$-axis and thus the plot has a constant "wavelength", not depending on $k$ like in Fig. 1. The envelope is given by:

$$
y=\frac{2 A}{(k+1)^{3 / 4}}, \quad A=0.777506276445256 \times 10^{-5}
$$

and was obtained in the following way: First we maintained in (16) only the first zero $\rho_{1}=1 / 2+\gamma_{1}$ :

$$
\begin{gathered}
\tilde{c}_{k}=\frac{2}{(k+1)^{3 / 4}} \times \\
\times\left(\alpha_{1} \cos \left(\frac{1}{2} \gamma_{1} \log (k+1)\right)-\beta_{1} \sin \left(\frac{1}{2} \gamma_{1} \log (k+1)\right)\right) .
\end{gathered}
$$

Next we made use of the identity:

$$
\begin{gathered}
a \cos (\theta)-b \sin (\theta)=A \sin (\phi-\theta) \quad \text { where } \\
A=\sqrt{a^{2}+b^{2}}, \quad \phi=\arctan \left(\frac{a}{b}\right)
\end{gathered}
$$

to obtain:

$$
\tilde{c}_{k}=\frac{2}{(k+1)^{3 / 4}} \sqrt{\alpha_{1}^{2}+\beta_{1}^{2}} \sin \left(\phi-\frac{1}{2} \gamma_{1} \log (k+1)\right) .
$$

from which (20) follows and a numerical value of $A$ is obtained from $\alpha_{1}$ and $\beta_{1}$ in Table 4. Here $\phi=-0.54916$ (=56.497 ). Let us remark that this value of $A$ agrees very well with the amplitude reported by Beltraminelli and Merlini [16]. It is interesting to note that lhs of the above formula is valid not only for integer $k$ but also for real $k$, thus using the approximation $c_{k} \approx R(k) / k$ derived in [10] we can write for large $x$ :

$$
\begin{gathered}
R(x)=2 x^{1 / 4} \times \\
\times\left(\alpha_{1} \cos \left(\frac{1}{2} \gamma_{l} \log (x)\right)-\beta_{1} \sin \left(\frac{1}{2} \gamma_{l} \log (x)\right)\right) .
\end{gathered}
$$

There is another way of checking accuracy of the above equation (23). Namely assuming that (23) is true and denoting by $k^{\prime}$ and $k$ ', two consecutive zeros of $c_{k}$, $=0$, $c_{k},=0$ we get for $\gamma_{1}$ :

$$
\gamma_{1}=\frac{2 \pi}{\log \left(\left(k^{\prime \prime}+1\right) /\left(k^{\prime}+1\right)\right)} .
$$

To make sense, in the latter approach an independent of (23) and relatively fast method of calculating $c_{k}$ is needed.
In fact in [4] Baez-Duarte gives among others following formula being the transformation of (1):

$$
c_{k}=\sum_{n=1}^{\infty} \frac{\mu(n)}{n^{2}}\left(1-\frac{1}{n^{2}}\right)^{k} .
$$

\begin{tabular}{|c|c|c|}
\hline & extrapolated zero $k^{\prime}$ & $\gamma_{1}$ from Eq. $(25)$ \\
\hline 1 & 19319.0191151 & 0.66394362155812867 \\
\hline 2 & 22526.0331312 & 40.91301517418643030 \\
\hline 3 & 41868.9707418 & 10.13657613078084811 \\
\hline 4 & 60094.3311655 & 17.38744852979003230 \\
\hline 5 & 98378.2514809 & 12.74744116303923005 \\
\hline 6 & 149320.1629630 & 15.05785832239914317 \\
\hline 7 & 236817.0977574 & 13.62376898016027559 \\
\hline 8 & 366000.4553802 & 14.43265811387491946 \\
\hline 9 & 573460.6753253 & 13.99205468779138406 \\
\hline 10 & 891841.3774543 & 14.22828231751450665 \\
\hline 11 & 1393469.2943691 & 14.07955314904129825 \\
\hline 12 & 2173554.0482344 & 14.13327453400666383 \\
\hline 13 & 3387835.5708183 & 14.15682029348025265 \\
\hline 14 & 5283842.8916393 & 14.13659885512530467 \\
\hline 15 & 8247263.1465316 & 14.11229699162269810 \\
\hline 16 & 12864372.4128156 & 14.13284964864925287 \\
\hline 17 & 20052822.4883780 & 14.15424390183719722 \\
\hline 18 & 31271608.9210745 & 14.14046804681939058 \\
\hline 19 & 48805962.1935733 & 14.11501826908551253 \\
\hline 20 & 76145459.4891092 & 14.12608973663948854 \\
\hline 21 & 118689214.0783221 & 14.15568694594979885 \\
\hline 22 & 185076299.0519995 & 14.14304477168657435 \\
\hline 23 & 288851950.0033488 & 14.11488330063446422 \\
\hline 24 & 450663149.7663923 & 14.12567920473796440 \\
\hline 25 & 702517003.8056590 & 14.15292988626078933 \\
\hline
\end{tabular}

Using this expression we have searched numerically for sign changes of $c_{k}$ up to $k=10^{9}$ and Table 5 presents $\gamma_{1}$ calculated from the above formula (25) together with consecutive zeros extrapolated from integer values using the linear approximation (let us remind that $\sin (x)$ has derivative \pm 1 at zeros!). The correct value is $\gamma_{1}=14.13472514173469379045725 \ldots$.

Table 5. 
In Fig. 2 the plot of $k^{3 / 4} c_{k}$ is presented. The Baez-Duarte criterion requires this "wave" to be contained in the strip of parallel lines for all $k$. Violation of the $\mathrm{RH}$ would manifest as an increase of the amplitude of the combination $k^{3 / 4} c_{k}$ for large $k$. This point is elaborated in more detail by Maślanka in [6]. Here we will make some further comments on this issue. First it should be remarked that the r.h.s. of (16) consists of products of three terms: the first depending only on $k$ (the overall factor $k^{3 / 4}$ ), the second depending only on imaginary parts of nontrivial zeros of $\zeta$ (the coefficients $\alpha_{l}$ and $\beta_{l}$ ) and third ingredients depending both on $k$ and $l$ (the trigonometric functions). Assume there are some zeros of $\zeta$ off critical line. We can split the sum over zeros $\rho$ in (11) in two parts: one over zeros on critical line and second over zeros off critical line. This second sum should violate the overall term $k^{-3 / 4}$ present in the first sum. Let $\gamma_{i}^{(o)}$ denote the imaginary parts of the zeros lying off critical line ("o" stands for "off"). It is not clear whether asymptotic similar to (19) will be valid for zeros off critical line, but it seems to be reasonable to assume that it should not differ significantly from (19). Then the contribution to $c_{k}$ of such zeros off critical line should contain a factor of the order $e^{-\gamma_{l}^{(o)}}$. Because the value of the imaginary part $\gamma_{l}^{(o)}$ of the hypothetical zero off critical line should be extremely large, perhaps even as large as $10^{100}$ can be expected, the combined contribution to $c_{k}$ coming from the second sum seems to be extremely small, thus to see violation of the Baez-Duarte criterion the values of $k$ should be larger than famous Skewes number and look something like $10^{10 .}$. Such a big index $k$ should cause that the first term in (16) overcame the smallness of the second term depending only on $\gamma_{l}^{(o)}$.

The plot in Fig. 2 is a perfect sine of one wavelength thus it gives visual justification of the above statement that $\tilde{c}$ is in fact determined by the first zero $\gamma_{1}$. The same phenomenon was mentioned by Maślanka in [6].

\section{FINAL REMARKS}

The formula (16) together with a few coefficients $\alpha_{l}$ and $\beta_{l}$ taken from Table 4 allows to compute values of $c_{k}$ for arbitrary large $k$. Other criteria for $\mathrm{RH}$, like the value of the de Bruijn-Newman constant [17], are vulnerable to the Lehmer pairs of zeros of $\zeta(s)$. It is hard to see the reason for violation of the inequality $\left|c_{k}\right|<$ const $k^{-3 / 4}$. We have checked that at the first Lehmer pair $\rho_{6709}=0.5+$ $7005.06286617 i$ and $\rho_{6710}=0.5+7005.1005646 i$ the derivative has value $\zeta_{i}^{\prime}(0.5+7005.06286617 i)=$ $3.2229849698+0.74179951875 i$ and a similar value for the second zero, thus there is no chance to get values of $c_{k}$ violating (2) in this way. It seems to be an open problem how to connect the value of the largest $k$ for which $\left|c_{k}\right|<$ const $k^{-3 / 4}$ to the number of zeros lying on the critical line. Let us mention that for the Li's criterion [18] which states that if the numbers

$$
\lambda_{n}=\left.\frac{1}{(n-1) !} \frac{d^{n}}{d s^{n}}\left(s^{n-1} \log \xi(s)\right)\right|_{s=1}
$$

fullill $\lambda_{n}>0$ for each $n$ then RH is true it is known that if the first $n$ Li's constants $\lambda_{n}$ are positive then every zero $\rho$ of $\zeta(s)$ with $|\mathfrak{I} \rho|<\sqrt{n}$ lies on the critical line $\Re \rho=1 / 2$ [19].

After a few months of computer experiments with $c_{k}$ we believe that Baez-Duarte sequence is one of the most important and mysterious sequences in the whole mathematics.

\section{Acknowledgement}

We thank Dr. J. Cisło for discussions and bringing to our attention identity (6), Prof. L. Baez-Duarte and Prof. K. Maślanka for e-mail exchange. The zeros of $\zeta(s)$ we have used in computations were taken from Prof. A. Odlyzko's web page http://www.dtc.umn.edu/ odlyzko/zeta_tables/index.html

\section{References}

[1] K. Maslanka, A hypergeometric-like Representation of Zeta-function of Riemann, Cracow Observatory preprint no. 1997/60, 1997; K. Maslanka, A hypergeometric-like Representation of Zeta-function of Riemann, posted at arXiv:math-ph/0105007 2001.

[2] L. Baez-Duarte, On Maslankas representation for the Riemann zeta function, 2003, math.NT/0307214.

[3 L. Baez-Duarte, A new necessary and su士cient condition for the Riemann Hypothesis, 2003, math.NT/0307215.

[4] L. Baez-Duarte, A sequential Riesz-like criterion for the Riemann Hypothesis, International Journal of Mathematics and Mathematical Sciences 3527-3537 (2005).

[5] K. Maślanka, Liczba $i$ kwant (in English: Number and quant), OBI 2004 Kraków.

[6] K. Maślanka, Baez-Duartes Criterion for the Riemann Hypothesis and Rices Integrals, math.NT/0603713 v2 1 Apr 2006.

[7] R. L. Graham, D. E. Knuth and O. Patashnik, Binomial Coefficients. Ch. 5 in Concrete Mathematics: A Foundation for Computer Science, 2nd ed. Reading, MA: AddisonWesley, 1994.

[8] E. C. Titchmarsh, The Theory of the Riemann Zeta Function, 2nd ed. New York: Clarendon Press, 1987.

[9] M. Riesz, Sur l'hypothe `se de Riemann, Acta Math. 40, 185-190 (1916). 
[10] J. Cislo and M. Wolf, Criteria equivalent to the Riemann Hypothesis, arXiv:math.NT/0808.0640v2.

[11] L. Baez-Duarte, Möbius-Convolutions and the Riemann Hypothesis, arXiv:math.NT/0504402

[12] PARI/GP, version 2.2.11, Bordeaux, 2005, http://pari.math.u-bordeaux.fr/.

[13] S. Beltraminelli and D. Merlini, The criteria of Riesz, Hardy-Littlewood et al. for the Riemann Hypothesis revisited using similar functions, math.NT/0601138.

[14] P. Flajolet and R. Sedgewick, Theoretical Computer Science, 144 (1-2), 101-124 (1995).

[15] E. C. Titchmarsh, The Theory of Functions, 2nd ed. Oxford, England: Oxford University Press, 1960.
[16] S. Beltraminelli and D. Merlini, Riemann Hypothesis: The Riesz-Hardy-Littlewood wave in the long wavelength region, math.NT/0605565

[17] A. M. Odlyzko, An improved bound for the de Bruijn-Newman constant, Numerical Algorithms 25, 293-303 (2000).

[18] X.-J. Li, The Positivity of a Sequence of Numbers and the Riemann Hypothesis, J. Number Th. 65, 325-333 (1997).

[19] J. Oesterle unpublished, cited in P. Biane, J. Pitman and M. Yor, Probability Laws Related to the Jacobi Theta and Riemann Zeta Functions, and Brownian Excursions. Bull. Amer. Math. Soc. 38, 435-465 (2001).

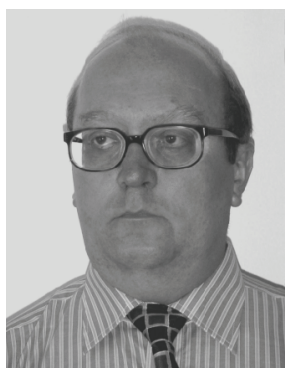

MAREK WOLF is a member of the Group of Mathematical Methods in Physics at the Institute of Theoretical Physics, University of Wroclaw. His M.Sc. in Physics (1978) thesis was devoted to the Nambu-Goto string, Ph.D. in Physics (1982) was about Central Charges in the Supersymmetric Quantum Field Theory. Since 1984, he has been doing computer experiments in physics and mathematics. 